\title{
Nose-only inhalation exposures to alumina nanoparticles / hydrogen chloride gas mixtures induce strong pulmonary pro-inflammatory
} response

\author{
Alexandra Bourgois \\ Institut de recherche biomedicale des armees \\ Dominique Saurat \\ Institut de recherche biomedicale des armees \\ Alexandre Boyard \\ Institut de recherche biomedicale des armees \\ Nathalie Guitard \\ Institut de recherche biomedicale des armees \\ Suzanne De Araujo \\ Institut de recherche biomedicale des armees
}

Sylvie Renault

Institut de recherche biomedicale des armees

Francisca Fargeau

Institut de recherche biomedicale des armees

Christine Frédéric

Institut de recherche biomedicale des armees

\section{Emmanuel Peyret}

Institut National de l'Environnement et des RISques

\section{Emmanuel Flahaut}

Universite Federale Toulouse Midi-Pyrenees

\section{Aurélie Servonnet}

Institut de recherche biomedicale des armees

Anne-Laure Favier

Institut de recherche biomedicale des armees

Ghislaine Lacroix

Institut National de l'Environnement industriel et des RISques

\section{Sabine François}

Institut de recherche biomedicale des armees

Samir Dekali ( $\nabla$ samir.dekali@gmail.com )

Institut de recherche biomedicale des armees

https://orcid.org/0000-0002-9662-0284 


\section{Research}

Keywords: pulmonary inflammation, mixtures, nose-only inhalation, combustion aerosols, alumina nanoparticles, hydrogen chloride

Posted Date: July 29th, 2020

DOI: https://doi.org/10.21203/rs.3.rs-41391/v2

License: (c) (1) This work is licensed under a Creative Commons Attribution 4.0 International License. Read Full License 
The authors have withdrawn this preprint from Research Square 